\title{
Unveiling the kinematics of the disk and the ionized stellar wind of the massive star MWC349A through RRL masers
}

\author{
Alejandro Báez-Rubio ${ }^{1}$ and Jesús Martín-Pintado ${ }^{2}$ \\ Centro de Astrobiología (CSIC-INTA), \\ Ctra de Torrejón a Ajalvir, km 4, 28850 Torrejón de Ardoz, Madrid, Spain \\ email: ${ }^{1}$ baezra@cab.inta-csic.es ${ }^{2}$ jmartin@cab.inta-csic.es
}

\begin{abstract}
The kinematics of photoevaporating disks and their associated ionized outflows around massive stars are fundamental to understand how these stars are formed and they evolve in their early phases of their evolution. To date, the important advances have been provided by studying the UC-HII region of MWC349A thanks to their strong maser emission at Hydrogen radio-recombination lines (RRLs). This $\mathrm{B}[\mathrm{e}]$ star is one of the best prototypes of massive star with an ionized outflow expanding at nearly constant velocity. A 3D radiative transfer model applied to the $\mathrm{H} 30 \alpha$ line has allowed to constrain the disk kinematics, which seems to follow pure Keplerian rotation in its outer parts. The model has also allowed us to constraints the launching radius of the outflow. Our results are supported by the agreement of our model predictions with the observations for other observed RRLs. Recent high-frequency observations of RRL masers with the Herschel Space Telescope (HIFI) show that the kinematics of the disk inner regions is not well understood. Modeling of these lines will constrain the formation of the ionized winds.
\end{abstract}

Keywords. accretion, accretion disks, masers, line: profiles, radiative transfer, stars: winds, outflows, radio continuum: stars, radio lines: stars, submillimeter

\section{Introduction}

Although massive stars play a central role in the evolution of the Cosmos, their short lifetime, their rarity and their large distances make their detailed study difficult. In particular, there is a lack in the understanding of how they form and their early and late stages of evolution. Recent studies have shown that at least in some cases, the formation of massive stars seems to proceed through accretion of circumstellar disks, as in low-mass stars (Jiménez-Serra et al. 2009, Kraus et al. 2010). On the other hand, the late stages of evolution contain a rich zoo of different kind of stars (i.e. LBV, WR). Some of them, such as the supergiant $\mathrm{B}[\mathrm{e}]$ stars, also have circumstellar disks. However, the kinematics of both the circumstellar disk and the ionized outflow are still poorly understood for both the pre-main or post-main sequence stars. One of the most relevant discoveries is the fact that UC-HII regions do not expand into the circumstellar medium as we could expect from the high pressure exerted by these hot ionized regions (Churchwell et al. 1990). Its explanation is still under debate. To unveil the kinematics of the ionized region around massive stars with circumstellar disks, we have studied the star of MWC349A due to its unique characteristics.

MWC349A is a star of spectral type B[e], characterized by its forbidden emission lines in the optical spectrum and its NIR excesses (Lamers et al. 1998). As often happens with $\mathrm{B}[\mathrm{e}]$ stars, we do not know its evolutionary stage. It could be a pre-main sequence star or a supergiant $\mathrm{B}[\mathrm{e}]$ star. Nevertheless, it is one of the few massive stars with a circumstellar disk well confirmed observationally and with an edge-on orientation very well established 
(Danchi et al. 2001). For years it has been the only source known with maser emission at Hydrogen RRLs as proved by the integrated line-to-continuum ratios (Martín-Pintado et al. 1989). The maser lines along with the fact that it is the strongest radio-continuum source provides spectra with a high angular and spatial resolution to study in detail the kinematics of its ionized wind. We have constrained the physical characteristics and kinematics of the ionized circumstellar disk by comparisons of the radio-continuum and RRL observations with the results of a non-LTE 3D radiative transfer model. Our results provide the first self-consistent model that explain the bulk of the available data.

\section{Radio-continuum emission}

First of all, we have constrained the physical characteristics and geometry for the ionized wind of MWC349A on the basis of the radio-continuum images and its spectral energy distribution. Its spectral index, $\sim 0.6$ (Tafoya et al. 2004), is explained as due to an ionized wind expanding at constant velocity, $v_{\text {exp }}$. We have explained those data by considering a double-cone geometry with semi-opening angle of $\theta_{a} \sim 57^{\circ}$, an electron density distribution depending on the radius and the zenith angle such as the density is larger at angles closer to the faces of the double-cone like $N_{e}(r, \theta)=3.85 \cdot 10^{9} e^{\frac{\theta_{a-\theta}-\theta}{20}}$. $r^{-2.14} \mathrm{~cm}^{-3}$ (with the angle $\theta$ as shown in Fig. 1) and an electron temperature of $12000 \mathrm{~K}$.

\section{RRL emission: constraining the kinematics of the ionized gas}

We have constrain the kinematics by using the whole data set of RRLs. The doublepeaked profiles and the $\mathrm{H} 30 \alpha$ centroid map show two kinematic components (see Fig. 1): an ionized Keplerian-rotating disk located next to the neutral disk with opening angle $\theta_{k}$ and an outflow that must be expanding at nearly constant velocity as previously mentioned. By modelling the source with these two kinematic components, we have obtained the following input parameters for the model: an inclination angle, $\theta_{i}$, of $8^{\circ}$ (tilted-up), an opening angle for the ionized rotating disk, $\theta_{k}$, of $6.5^{\circ}$ which extends up $130 \mathrm{AU}$, a central mass for the star of $40 M_{\odot}$, an electron temperature of $10000 \mathrm{~K}$ for the disk, a terminal velocity of $60 \mathrm{~km} \mathrm{~s}^{-1}$ for the outflow and a turbulent velocity of $15 \mathrm{~km} \mathrm{~s}^{-1}$.

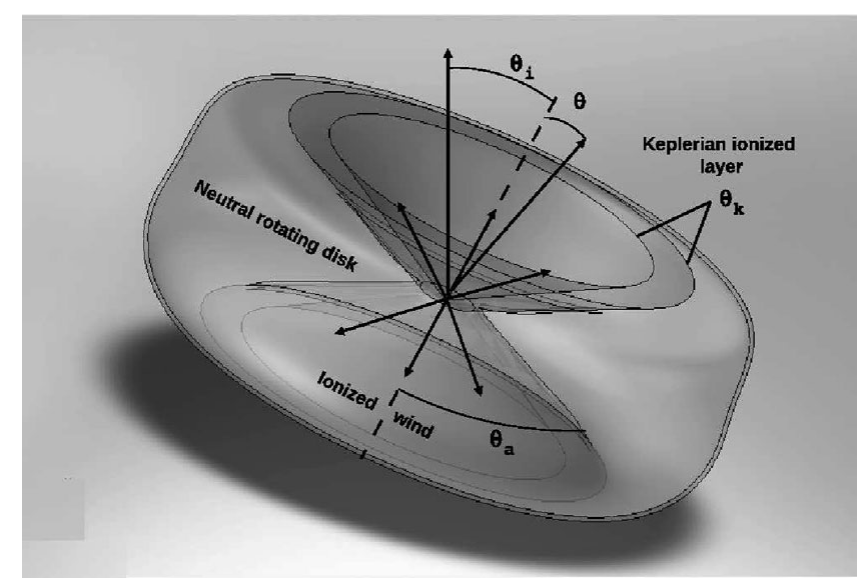

Figure 1. Sketch of the double-cone geometry used for the modelling the Keplerian-rotating ionized disk and the outflow of MWC349A. 


\section{1. $H 30 \alpha$ centroid map}

The interpretation that the UC-HII region of MWC349A is composed by an ionized rotating disk and an outflow is supported by the behaviour of the H30 $\alpha$ centroid map (see Fig. 2). The centroid map provides the spatial location where the line emission arises for every radial velocity with a relative accuracy of $\sim 2$ to 5 milliarcseconds (Martín-Pintado et al. 2011).

In the centroid map we clearly distinguish two different regions. Firstly, a straight line overlapping with the NIR disk image (Danchi et al. 2001) for the radial velocities between the maser spikes. Our model clearly shows that the line emission for that range of radial velocities is mainly originated inside of the disk. On the other hand, the expanding outflow is responsible for the north-south loops observed in the centroid map for radial velocities larger than those of the maser spikes.

Since the intensity of the maser emission strongly depends on the electron density and electron temperature (Strelnitski et al. 1996), it provides tight constraints on the kinematics of the region where the maser spikes arise. By using the velocity peak separations and the $\mathrm{H} 30 \alpha$ centroid map, we have deduced that the circumstellar disk is rotating following a Keplerian law around a central star of about $\sim 40 \mathrm{M}_{\odot}$.

On the other hand, since the emission of the outflow should be symmetric with respect the East-West plane for an edge-on disk, the north-south loops observed in the H30 $\alpha$ centroid map must be due to the fact that the disk is slightly tilted. The fit to the height of the loops provides strong constraints on the inclination of the plane of the disk with respect the line-of-sight: between 4.5 and $15^{\circ}$ for a tilted-up disk. In addition, the loops are a clear signature that the outflow is also rotating since otherwise, the emission would be symmetrical with respect to the north-south plane. In such a case, the loops would occur at right ascensions very close to 0 (Martín-Pintado et al. 2011), opposite to that observed. Thus, our model predicts the rotation for the outflow following the Keplerian law of the ionized circumstellar disk.

Another important finding is that the $\mathrm{H} 30 \alpha$ centroid map is only explained if we assume that the ionized outflow is originated in the inner disk at a radius of $<25 \mathrm{AU}$. This fact clearly disagrees with the more than 100 AU expected from the analytic photoevaporating model of Hollenbach et al. (1994) and it could provide constraints on which hydrodynamical models are viable candidates to explain the formation of the ionized

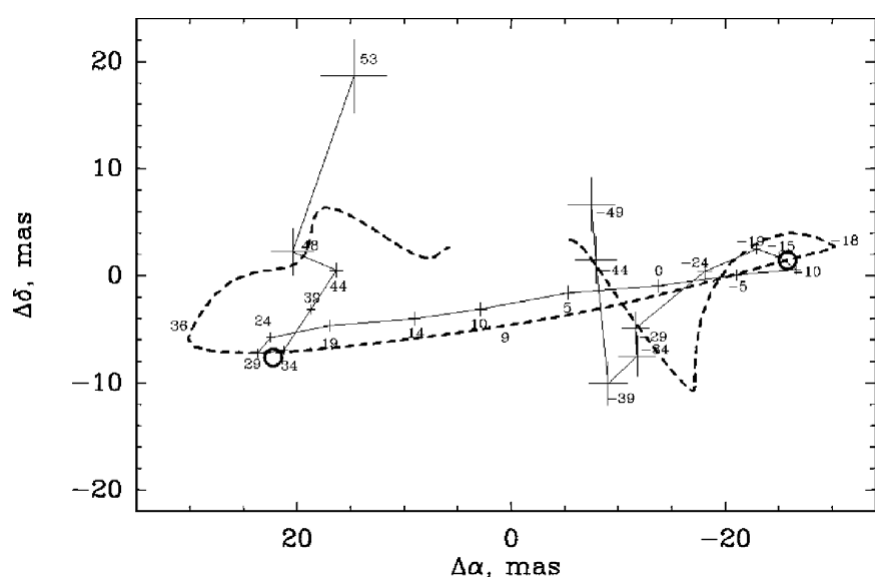

Figure 2. Observational (solid lines) and predicted (dashed lines) centroid map of the H30 $\alpha$. The observations were carried out with the IRAM Plateau de Bure Interferometer (Martín-Pintado et al. 2011). 
Finally we have to stress that our final results were obtained by using the set of departure coefficients, $b_{n}$, provided by Storey \& Hummer (1995) since no one set of input parameters reproduce the intensity of the maser spikes of mm RRLs if we use the set of $b_{n}$ provided by Walmsley (1990). In particular, our model reproduces for the first time the intensities of the maser spikes at $\mathrm{mm}$ wavelengths and their integrated line fluxes.

\subsection{Integrated line fluxes}

The non-LTE model predictions matches well the observed integrated line fluxes for Hn $\alpha$ with $n>26$ as shown in Fig. 3. This supports the proposed kinematic model and gives hints on the dominant processes involved in the RRL emission for the different ranges of frequencies.

Our predictions show that even for low frequencies RRLs (those with $n>41$ ), there is a constant shift between the non-LTE and the LTE predictions. This seems to indicate that even for such RRLs, the emission is out of the LTE since the observations can only be fitted by considering the non-LTE case. This is also consistent with the profile found for the observed RRL of largest frequency, the H76 $\alpha$ (see Escalante et al. 1989). Its asymmetric profile is easily explained due to stimulated emission. In particular, the stronger emission of the blue-shifted wing is due to the amplification of the larger background continuum emission by the ionized material approaching to us. All these evidences seem to indicate that the radiation is amplified by stimulated emission even for low-frequency RRLs.

Secondly, the integrated line fluxes for Hn $\alpha$ RRLs with $21<\mathrm{n}<41$ significantly increases with the frequency, specially for RRLs with $\mathrm{n}<31$. In this range of RRLs, the maser emission is the dominant process. Our non-LTE predictions agree very well with the predictions down to the H26 $\alpha$. However, we clearly overestimate the integrated line flux of the submm RRLs with $\mathrm{n}<26$. This is because in our model the maser amplification increases in an exponential way for such RRLs, while one expects that such strong maser emission should be saturated. Thus, we would need to take into account in our model the saturation effects to explain the intensities of those lines. Finally our LTE predictions show that the RRL emission are emitted under LTE conditions for $n<7$.

\subsection{The inner disk kinematics. Keplerian rotation?}

The increase of the velocity peak separation of the maser spikes with frequency is well explained by our model for millimeter RRLs for frequencies larger than that of the H30 .

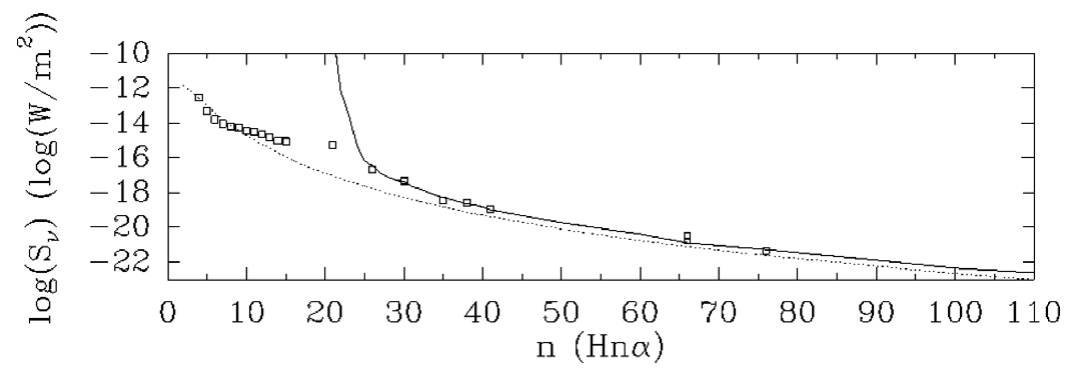

Figure 3. Observational (squares) and predicted integrated line fluxes for the non-LTE and LTE case (solid and dashed lines respectively). References for the observational data: H4 $\alpha$ to

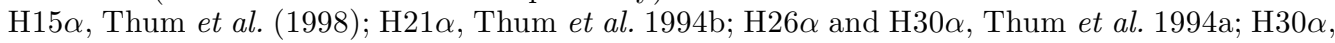

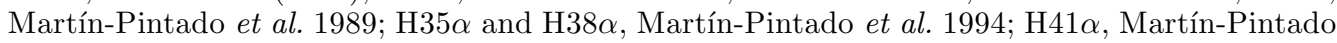
et al. 1989; H66 $\alpha$, Loinard \& Rodríguez 2010; H66 $\alpha$, Martín-Pintado et al. 1993; H76 $\alpha$, Escalante et al. 1989. 
Nevertheless, the H26 $\alpha$ and H21 $\alpha$ profiles obtained with the IRAM-30m (Thum et al. 1995, Thum et al. 1994 respectively) and the very recent observations carried out provided by the HIFI instrument on board the Herschel Space Observatory show that the velocity peak separation does not increase as expected from the model for a Keplerian-rotating disk. In contrast, it remains approximately constant $\left(\sim 50 \mathrm{~km} \mathrm{~s}^{-1}\right)$ from the $\mathrm{H} 24 \alpha$ to the H16 $\alpha$. The explanation of this behaviour will help to understand the inner regions of the disk, where the outflow is expected to originate. It could be a signature of the distortion of the disk kinematics of the inner regions likely due to launching of the ionized outflow.

\section{Summary and perspectives}

For the first time, we have used the powerful maser emission of hydrogen RRLs to derive the detailed kinematics of the ionized circumstellar disk and the ionized outflow around a massive star. By using the information derived from the maser RRLs along with the data of other RRLs, we have presented the first self-consistent model that describes the bulk of the observations carried out in a wide range of frequencies. Future submm high angular studies will help understanding the kinematics of the inner regions and the possible the mechanisms involved in the launching of the outflow. In addition, the recent detection of pre-main sequence stars with RRL maser emission (Jiménez-Serra et al. 2011, Jiménez-Serra et al. in prep.) open the possibility that RRL maser emission turns out to be an essential tool to disentangle the kinematics of circumstellar disks around massive stars.

\section{Acknowledgements}

We thank the Spanish MICINN for funding support through grants ESP2007-65812C02-C01 and AYA2010-21697-C05-01 and AstroMadrid (CAM S2009/ESP-1496). A. Báez-Rubio acknowledges support from grant JAE program, CSIC, Spain.

\section{References}

Churchwell, E. 1990, A\& $\&$ R, 2, 79

Danchi, W. C., Tuthill, P. G., \& Monnier, J. D. 2001, ApJ, 562, 440

Escalante, V., Rodríguez, L. F., Moran, J. M., \& Cantó, J. 1989, Rev. Mexicana AyA, 17, 11

Hollenbach, D., Johnstone, D., Lizano, S., \& Shu, F. 1994, ApJ, 428, 654

Jiménez-Serra, I., Martín-Pintado, J., Caselli, P., Martín, S. et al. 2009, ApJ, 703, L157

Jiménez-Serra, I., Martín-Pintado, J., Báez-Rubio, Al. et al., 2011 ApJ, 732, L27

Kraus, S., Hofmann, K., Menten, K. M., Schertl, D., Weigelt, G. et al. 2010, Nature, 466, 339

Lamers, H. J. G. L. M., Zickgraf, F.-J., de Winter, D. et al. 1998, A\&\&A 340, 117

Loinard, L. \& Rodríguez, L. F. 2010 ApJ, 722, L100

Martín-Pintado, J., Bachiller, R., Thum, C., \& Walmsley, M. 1989, A\&3A, 215, L13

Martín-Pintado, J., Gaume, R., Bachiller, R., Johnston, K., \& Planesas, P. 1993, ApJ, 418, L79

Martín-Pintado, J., Neri, R., Thum, C., Planesas, P., \& Bachiller, R. 1994, A\&A, 286, 890

Martín-Pintado, J., Thum, C., Planesas, P., \& Báez-Rubio, A. 2011, A\&\&A, 530, L15

Storey, P. J. \& Hummer, D. G. 1995, MNRAS, 272, 41

Strelnitski, V. S., Ponomarev, V. O., \& Smith, H. A. 1996, ApJ, 470, 1118

Tafoya, D., Gómez, Y., \& Rodríguez, L. F. 2004, ApJ, 610, 827

Thum, C., Matthews, H. E., Martín-Pintado, J. et al. 1994, A\& A, 283, 582

Thum, C., Matthews, H. E., Harris, A. H., Tacconi, L. J. et al. 1994, A\&\&A, 288, L25

Thum, C., Strelnitski, V. S., Martín-Pintado, J. et al. 1995, A\&SA, 300, 843

Thum, C., Martín-Pintado, J., Quirrenbach, A. \& Matthews, H. E. A\&A, 333, L63

Walmsley, C. M. 1990, AESA, 82, 201 